\title{
Relationship Between Colon Wall Thickness in Computed Tomography Scan and Colon Cancer: A Retrospective Study
}

\author{
(1) Önder Karabay1, (1) Nadir Adnan Hacım², (1) Mert Gençtürk³ \\ ${ }^{1}$ Yedikule Surp Pirgiç Armenian Hospital, Clinic of General Surgery, İstanbul, Turkey \\ 2University of Health Sciences Turkey, Bağcılar Training and Research Hospital, Clinic of General Surgery, İstanbul, Turkey \\ ${ }^{3}$ Yedikule Surp Pirgiç Armenian Hospital, Clinic of Radiology, İstanbul, Turkey
}

Cite this article as: Karabay Ö, Hacım NA, Gençtürk M. Relationship Between Colon Wall Thickness in Computed Tomography Scan and Colon Cancer: A Retrospective Study. J Acad Res Med 2021;11(1):46-50

\begin{abstract}
Objective: This study aimed to investigate the colonoscopic findings of patients with increased colonic wall thickness (ICWT) detected by abdominal computed tomography (CT) and to define the effectiveness of neutrophil/lymphocyte ratio (NLR), platelet/lymphocyte ratio (PLR) and mean platelet volume (MPV) together with colonic wall thickness in predicting malignancy.

Methods: Data of patients who underwent abdominal CT for any reason between January 2017 and August 2019 were retrospectively reviewed. Records of patients with ICWT in the abdominal CT report were retrospectively analysed. Patients whose colon wall thickness could be measured and who were evaluated with colonoscopy and biopsy within 1-3 months after CT were included in the study. Haemoglobin (hb), albumin, NLR, PLR and MPV values, colon wall thickness and colonoscopy and biopsy results were recorded.

Results: Ninety-seven patients had ICWT data on CT. The colonic wall thickness and presence of positive lymph node were significantly higher in the malignancy group $(p<0.001)$. Similarly, values of $h b, N L R, P L R$ and MPV were different in the malignancy group ( $p<0.001)$. According to the receiver operating characteristics analysis, colon wall thickness over the $8.5 \mathrm{~mm}$ threshold value was a significant factor in predicting colon cancer ( $<<0.001)$.

Conclusion: In patients who do not have a history of gastrointestinal injury or disease, the incidental detection of ICWT $>8.5 \mathrm{~mm}$ may be an important finding for a possible diagnosis of colon cancer.
\end{abstract}

Keywords: Colonoscopy, colonic wall thickening, computed tomography, neutrophil lymphocyte ratio, platelet lymphocyte ratio

\section{INTRODUCTION}

Computed tomography (CT) is one of the most commonly used radiological imaging methods in the diagnosis of gastrointestinal diseases. Detection of increased colon wall thickness (ICWT) on CT is regarded as an important finding that necessitates additional evaluation (1). Although ICWT may be an imaging finding of colon cancer, it may occur because of several reasons such as peristaltism, insufficient filling of the colon lumen, faecal fragments and inflammatory gastrointestinal diseases. However, it can also be seen in some systemic conditions including cirrhosis, heart failure and hypoalbuminaemia (2).

When ICWT is detected on CT, examination of the lumen by colonoscopy is often requested. However, complications related to endoscopic procedures and an increase in cost are other

ORCID IDs of the authors: Ö.K. 0000-0002-3797-0102; N.A.H. 0000-0002-3906-2538; M.G. 0000-0001-9866-4312.

Corresponding Author/Sorumlu Yazar: Önder Karabay, E-mail: onderkarabay@gmail.com
Received Date/Geliş Tarihi: 01.10.2020 Accepted Date/Kabul Tarihi: 19.01.2021

(C) Copyright 2021 by University of Health Sciences Turkey, Gaziosmanpaşa Training and Research Hospital. Available on-line at www.jarem.org 
problems. In addition, the presence of a normal colonoscopy in some patients leads to the questioning of $\mathrm{CT}$ findings retrospectively. Besides, no guideline stated that colonoscopy should be performed when ICWT is seen during CT (1).

Few studies have focused on the relationship between ICWT and colonic diseases (3). Previous studies have speculated that a relationship exists between the degree of increased wall thickness in $\mathrm{CT}$ and colon cancer. Studies have also shown that the possibility of colon cancer increases if ICWT is associated with a mass $(4,5)$. Algorithms on ICWT's association with other findings such as changes in pericolonic fatty tissues and presence of lymph nodes have been studied (6). However, the correlation between isolated colonic wall thickness and colon cancer remains controversial.

Recent studies have extensively investigated the relationship between neutrophil/lymphocyte ratio (NLR), platelet/lymphocyte ratio (PLR) and mean platelet volume (MPV) values with tumoural and inflammatory diseases (7-10). NLR, PLR and MPV are thought to have diagnostic and prognostic roles in patients with colon cancer (7-10). In patients with ICWT, evaluating these laboratory values together with $C T$ findings may be useful to increase diagnostic efficiency.

This study aimed to investigate the colonoscopic findings of patients with ICWT detected by abdominal CT and to determine the effectiveness of NLR, PLR and MPV with colonic wall thickness in predicting malignancy.

\section{METHODS}

Patients who underwent abdominal CT for any reason between January 2017 and August 2019 were retrospectively reviewed. The phrase "ICWT" was searched for in CT reports. The study was conducted in accordance with the principles of the Declaration of Helsinki. Informed consent was obtained from all patients. Institutional approval was obtained due to the retrospective design of the study (Surp Pirgiç Armenian Hospital, 2228/2020).

\section{Study Design}

Records of patients with "ICWT" in the abdominal CT report were retrospectively analysed. Patients with oral and intravenous contrast-enhanced CT protocols whose colon wall thickness could be measured and who were evaluated with colonoscopy and biopsy within 1-3 months after CT were included in the study. The following conditions that interfered with optimal measurement of the colon wall thickness were excluded: Patients who do not comply with abdominal CT imaging protocols, who do not develop sufficient distension in the colon, who have solid-liquid stool in the colon, who have heart failure, hypoalbuminaemia and nephrotic syndrome that may affect the colon wall thickness, who have a history of abdominal surgery, who were followed up due to any type of bowel diseases, whose CT findings suggested colon cancer, and who had not undergone colonoscopy or had insufficient colonoscopy were excluded from the study. A total of 97 patients who met the study criteria at the last evaluation were included in the study (Figure 1).
Demographic data, haemoglobin [(hb), g/dL], albumin (g/dL), NLR, platelet PLR and MPV (f/L) values, colon wall thickness measurement $(\mathrm{mm})$, colonoscopy and biopsy results of the patients were recorded.

\section{Groups}

Abdominal CT images of the cases included in the study were reevaluated by an experienced radiologist who was unaware of the results of the colonoscopic-histopathological evaluation. ICWT over $3 \mathrm{~mm}$ was considered pathological (11). Cases were divided into three groups as normal, benign or malignant according to the results of the colonoscopic evaluation. The first group included patients who had normal colonoscopic findings and did not require further examination, and the second group included benign lesions of the colon that were not considered malignant by colonoscopy. Patients diagnosed with colonic inflammatory causes, diverticula and polyps by endoscopist were included in this group. The third group included patients with lesions diagnosed as malignant colonoscopically.

Cases were divided into two groups as benign and malignant according to their definitive pathological results. In the malignant

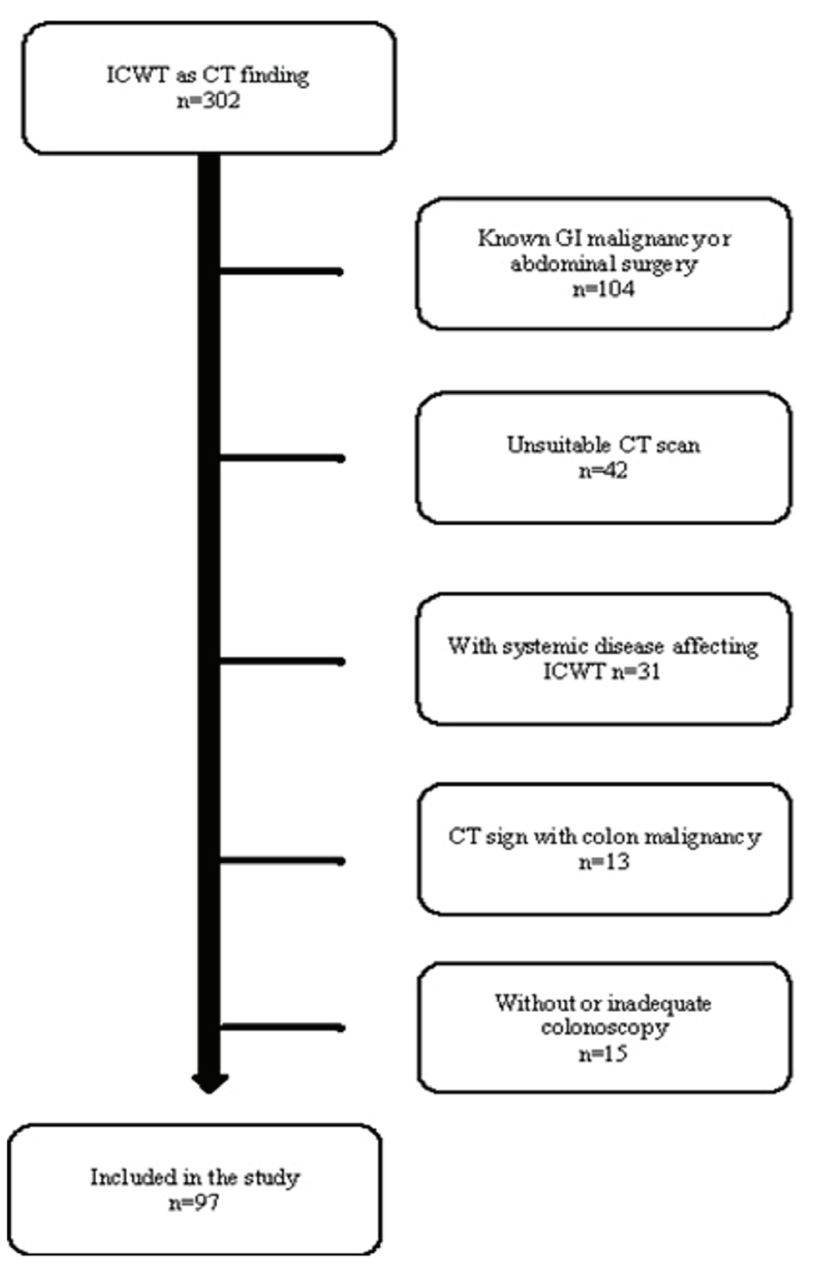

Figure 1. Flowchart of the study ICWT: increased colonic wall thickness, CT: computed tomography, Gl: gastrointestinal system 
group, patients had colon cancer proven pathologically. In the benign group, patients had normal pathology and patients with other benign lesions of the colon other than cancer.

\section{Statistical Analysis}

According to the results of colonoscopy and pathological examination, the difference between the groups in terms of the degree of ICWT, age, hb, albumin, NLR, PLR and MPV was investigated with descriptive statistics. Descriptive analysis was made to give information about the general characteristics of the study groups. Continuous variables were presented as mean \pm standard deviation or median ( $25^{\text {th }}-75^{\text {th }}$ percentile) depending on their normal distribution pattern. Data on categorical variables were given as frequency with percentage. While comparing the means of quantitative variables between groups, the significance test of the difference between two means and One-Way analysis of variance were used. Tukey honestly significant difference test was used for multiple comparisons to evaluate the groups responsible for the significant difference. Cross tables were created for qualitative variables, and chi-square tests were used for relationships between relevant variables.

The receiver operating curve (ROC) analysis associated with the area under the curve was used to determine optimal threshold values of colonic wall thickness in predicting the presence of pathologically proven colon cancer. Subsequently, patients were analysed as low or high groups according to threshold values to evaluate the relationship between the increase in colon wall thickness and presence of colon cancer. $\mathrm{P}<0.05$ value was considered statistically significant. Statistical analysis was performed with IBM SPSS 19 (IBM Corp., Armonk, NY) software.

\section{RESULTS}

Of the 97 patients, 59 (60.8\%) were male and 38 (39.2\%) were female. The mean age was $62.1 \pm 13$ years. When classified according to localisation, ICWT was seen mostly in the left colon (63.9\%). The median wall thickness of the colon was 9 (7-11) $\mathrm{mm}$. Positive lymph node was observed on CT of 25 (25.7\%) patients.

After colonoscopic evaluation, 31 patients (32.0\%) were classified into group 1, 39 (40.2\%) into group 2 (22 polyps, 9 colitis, 8 diverticular diseases) and 27 (27.8\%) (adenocarcinoma) into group 3. No significant difference was found between the groups in terms of age and sex ( $p>0.05)$. The median wall thickness of the colon were 9 (7-10) $\mathrm{mm}$ in group 1, 8 (7-9.5) $\mathrm{mm}$ in group 2 and 10 (9-14.5) $\mathrm{mm}$ in group 3 (Table 1). While no significant difference was noted between groups 1 and 2 in terms of the degree of

\begin{tabular}{|c|c|c|c|}
\hline & $\begin{array}{l}\text { Group } 1 \\
(n=31)\end{array}$ & $\begin{array}{l}\text { Group } 2 \\
(n=39)\end{array}$ & $\begin{array}{l}\text { Group } 3 \\
(n=27)\end{array}$ \\
\hline $\begin{array}{l}\text { CWT (mm) } \\
\left(25^{\text {th }}-75^{\text {th }} \text { percentile) }\right.\end{array}$ & $9(7-10)$ & $9(7-9.5)$ & $10(9-14.5)$ \\
\hline Pathologic LAP, n (\%) & $2(6.5 \%)$ & 5 (12.8\%) & 18 (66.7\%) \\
\hline
\end{tabular}

ICWT, the wall thickness in group 3 was significantly higher than that of group 1 and group $2(p=0.001)$.

When the three groups were compared based on the colonoscopic diagnosis, two (6.5\%) and five patients (12.8\%) in groups 1 and 2 had positive lymph nodes, respectively, whereas positive lymph nodes were detected in 18 patients $(66.7 \%)$ in group 3 . The rate of positive lymph node was significantly higher in group 2 than in other groups $(p=0.001)$.

According to the pathology results, when malignant and benign groups were compared in terms of colon wall thickness, the ICWT value was significantly higher in the malignancy group $(p=0.001)$.

The values of hb NLR, PLR and MPV were significantly different in group 3 than in groups 1 and $2(p<0.001)$. However, no difference was noted between the groups in terms of the serum albumin value.

ROC analysis using sensitivity and specificity to determine threshold values based on pathological colon cancer diagnosis revealed that the optimal threshold value for colon wall thickness

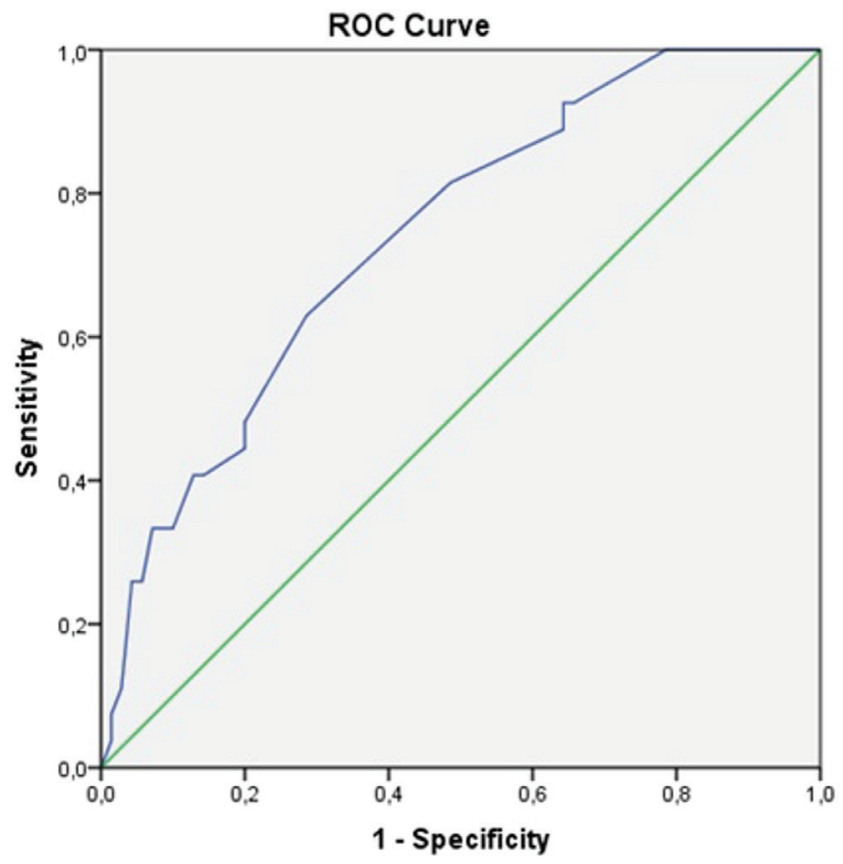

Figure 2. Receiver operating characteristics curve ROC: receiver operating characteristics

Table 2. Comparison of groups according to the threshold value determined according to the result of the ROC analysis

\begin{tabular}{l|l|l|} 
& $\begin{array}{l}\text { CWT <8.5 } \mathbf{~ m m} \\
(\mathbf{n}=\mathbf{4 1})\end{array}$ & $\begin{array}{l}\text { CWT } \geq 8.5 \mathrm{~mm} \\
(\mathbf{n = 5 6 )}\end{array}$ \\
\hline $\begin{array}{l}\text { CWT }(\mathrm{mm}) \\
\left(25^{\text {th }}-75^{\text {th }} \text { percentile) }\right.\end{array}$ & $7(6-8)$ & $10(9-13.7)$ \\
\hline $\begin{array}{l}\text { Pathologic LAP, n (\%) } \\
\text { Patient with malignancy (n) }\end{array}$ & 5 & 20 \\
\hline $\begin{array}{l}\text { CWT: colonic wall thickness, LAP: lymphadenopathy, ROC: receiver operat- } \\
\text { ing characteristics }\end{array}$ & 5
\end{tabular}


was $8.5 \mathrm{~mm}$. Optimal threshold sensitivity and specificity values are shown in Figure 2.

In the grouping according to the $8.5 \mathrm{~mm}$ threshold value, 56 patients (57.7\%) had ICWT. In these patients, 22 (39.2\%) had colon cancer and 20 (35.7\%) had positive lymph nodes. The presence of colon cancer and positive lymph nodes on CT were significantly higher in patients with ICWT ( $p=0.001$ ) (Table 2).

\section{DISCUSSION}

This study shows that ICWT of $>8.5 \mathrm{~mm}$ as an abdominal CT finding in patients who had not had gastrointestinal system disease or surgery before could be associated with a possible diagnosis of colonic malignancy.

Although ICWT in CT is not a specific finding, it may be a symptom of colon diseases, including colon cancer. The importance of the incidentally detected ICWT is not completely clear (4). There is no algorithm for the management of these patients. If an appropriate colonic lumen expansion can be achieved during $C T$, normal colonic wall thickness should not be $>3 \mathrm{~mm}$ (12).

According to the literature, colonoscopy is regarded as normal at the rates varying between $12 \%$ and $28 \%$ of patients with ICWT $(3,4,11,13-16)$. In this study, colonoscopy results were normal in $31.9 \%$ of the patients with ICWT. This means the additional cost and complication risk of colonoscopy in nearly one-third of the patients. On the contrary, the same studies have observed that the rate of colon cancer in patients with ICWT ranges from $14 \%$ to $46.5 \%(4,11,13-16)$. In our study, similar to the literature, the rate of colonic malignancy was $27.8 \%$.

Another CT finding of colon cancer is the presence of enlarged lymph nodes in the region adjacent to the related colon loops. In the colon cancer group, $66.7 \%$ of the patients had positive lymph nodes, which was significantly higher than those in the groups with normal and benign diseases.

Few studies have examined the amount of ICWT and colonoscopy findings $(3,14)$. According to Ergul and Filik (3), the average ICWT values were $8 \mathrm{~mm}$ and $15 \mathrm{~mm}$ in the normal and malignant groups, respectively. The mean values were 9.4 and $16.2 \mathrm{~mm}$ in Akbas et al.'s (14) study. In both studies, the thickness measurement was significantly higher in the malignancy group. In our study, ICWT values were $8.6 \mathrm{~mm}$ and $11.5 \mathrm{~mm}$ in the normal and malignancy groups, respectively. To distinguish patients with benign pathologies and to avoid unnecessary colonoscopy, a threshold value of $8.5 \mathrm{~mm}$ was determined for the wall thickness in patients with ICWT by ROC analysis. When grouping according to this threshold value, the rate of normal colonoscopy results decreased to $28.5 \%$. We believe that such threshold values are necessary in prospective larger groups in association with additional CT findings to decrease the need for colonoscopy.

Some parameters known to increase in inflammatory processes are thought to be linked to colon cancer (7-10). If inflammatory markers such as NLR, PLR, hb and MPV are associated with ICWT, they may be associated with colon cancer. In our study, these laboratory markers were significantly different in the malignancy group. A significant relationship was found between their association with ICWT and suspicion of colon cancer. This suggests that colonoscopy request can be determined more effectively using these tests.

\section{Study Limitations}

The limitations of our study were the small number of patients and the retrospective study design. More efficient results can be obtained with multicentre prospective studies with a large number of patients.

\section{CONCLUSION}

As a result, in patients who do not have a history of gastrointestinal surgery or disease, an incidental detection of ICWT $>8.5 \mathrm{~mm}$ may be important for a possible diagnosis of colon cancer. Besides, positive lymph nodes in association with ICWT might be a more predictive finding for the detection of colonic cancer. Colonoscopic examinations should be taken into consideration in patients with these findings.

Ethics Committee Approval: Institutional approval was obtained due to the retrospective design of the study (Surp Pirgic Armenian Hospital, 2228/2020).

Informed Consent: Informed consent was obtained from all patients.

Peer-review: Externally and internally peer-reviewed.

Author Contributions: Surgical and Medical Practices - Ö.K., N.A.H., M.G.; Concept - Ö.K., M.G.; Design - Ö.K., N.A.H.; Data Collection and/ or Processing - Ö.K., N.A.H., M.G.; Analysis and/or Interpretation - Ö.K., N.A.H., M.G.; Literature Search - Ö.K., M.G.; Writing - Ö.K., N.A.H.

Conflict of Interest: The authors have no conflict of interest to declare.

Financial Disclosure: The authors declared that this study has received no financial support.

\section{REFERENCES}

1. Chandrapalan $S$, Tahir F, Sinha R, Arasaradnam R. Colonic thickening on computed tomography-does it correlate with endoscopic findings? A protocol for systematic review. Syst Rev 2016; 5: 213.

2. Macari M, Balthazar EJ. CT of bowel wall thickening: significance and pitfalls of interpretation. AJR Am J Roentgenol 2001; 176: 1105-16.

3. Ergul B, Filik L. Clinical approach to gastrointestinal wall thickening: $A$ prospective single center study. Endoscopy 2012; 20: 29-31.

4. Moraitis $D$, Singh $P$, Jayadevan $R$, Cayten CG. Colonic wall thickening on computed tomography scan and clinical correlation. Does it suggest the presence of an underlying neoplasia? Am Surg 2006; 72: 269-71.

5. Padda M, Vadgama J, Sandhu P, Dev A, Giannikopoulos I. Clinical significance of incidental colorectal wall thickening on computed tomography scan in African- American and Hispanic patients. Dig Dis Sci 2007; 52: 3159-64.

6. Fernandes T, Oliveira MI, Castro R, Araujo B, Viamonte B, Cunha R. Bowel wall thickening at CT: simplifying the diagnosis. Insights Imaging 2014; 5: 195-208.

7. Walsh S, Cook E, Goulder F, Justin T, Keeling N, Neutrophil-lymphocyte ratio as a prognostic factor in colorectalcancer. J Surg Oncol 2005; 91: 181-4.

8. Kwon HC, Kim SH, Oh SY, Lee S, Lee JH, Choi HJ, et al. Clinical significanceof preoperative neutrophil-lymphocyte versus plateletlymphocyte ratio in patients with operable colorectal cancer. Biomarkers 2012; 17: 216-22.

9. Oflazoglu U, Alacacioglu A, Somali I, Yuce M, Buyruk AM, Varol U, et al. Prognostic value of neutrophi//lymphocyte ratio (NLR), platelet/ 
lymphocyte ratio (PLR) and mean platelet volume (MPV) in patients with colorectal carcinoma [lzmir Oncology Group (IZOG) study]. Acta Oncol Tur 2017; 50: 1-6.

10. Anuk T, Cığsar G, Yıldırım AC, Özgehan G, Gökce E, Güzel H, et al. Platelet count and mean platelet volume in the prediction of colorectal cancer in patients presented with emergency ileus. Kafkas J Med Sci 2015; 5: 65-9.

11. Nicholson BD, Hyland R, Rembacken BJ, Denyer M, Hull MA, Tolan DJ. Colonoscopy for colonic wall thickening at computed tomography: a worthwhile pursuit? Surg Endosc 2011; 25: 2586-91.

12. Fisher J. Abnormal colonic wall thickening on computed tomography. J Comput Assist Tomogr 1983; 7: 90-7.
13. Wolff JH, Rubin A, Potter JD, Lattimore W, Resnick MB, Murphy BL, et al. Clinical significance of colonoscopic findings associated with colonic thickening on computed tomography: is colonoscopy warranted when thickening is detected? J Clin Gastroenterol 2008; 42: 472-5.

14. Akbas A, Bakir H, Dasiran MF, Dagmura H, Daldal E, Ozsoy Z, et al. Colonic wall thickening reported in abdominal CT: does it always imply malignancy? Gastroenterol Res Pract 2019; 2019: 2492097.

15. Troppmann M, Lippert E, Hamer OW, Kirchner G, Endlicher E. Colonic bowel wall thickening: is there a need for endoscopic evaluation? Int J Colorectal Dis 2012; 27: 601-4.

16. Iadicola D, De Marco P, Bonventre S, Grutta EM, Barletta G, Licari L, et al. Bowel wall thickening: inquire or not inquire? Our guidelines. G Chir 2018; 39: 41-4. 